\title{
EchoGéo
}

\section{La diversification des trajectoires de métropolisation au sud du Vietnam}

L'exemple de la mise en concurrence entre Hô Chi Minh Ville et Binh Duong

Diversification of metropolization trajectories in southern Vietnam. Exemple of the competition between Ho Chi Minh City and Binh Duong.

\section{Khac Minh Tran}

\section{CpenEdition}

Journals

Édition électronique

URL : https://journals.openedition.org/echogeo/19506

DOI : $10.4000 /$ echogeo. 19506

ISSN : 1963-1197

Éditeur

Pôle de recherche pour l'organisation et la diffusion de l'information géographique (CNRS UMR 8586)

Référence électronique

Khac Minh Tran, «La diversification des trajectoires de métropolisation au sud du Vietnam », EchoGéo [En ligne], 52 | 2020, mis en ligne le 30 juillet 2020, consulté le 28 juin 2022. URL : http:// journals.openedition.org/echogeo/19506; DOI : https://doi.org/10.4000/echogeo.19506

Ce document a été généré automatiquement le 11 août 2021.

EchoGéo est mis à disposition selon les termes de la licence Creative Commons Attribution - Pas d'Utilisation Commerciale - Pas de Modification 4.0 International (CC BY-NC-ND) 


\title{
La diversification des trajectoires de métropolisation au sud du Vietnam
}

\author{
L'exemple de la mise en concurrence entre Hô Chi Minh Ville et \\ Binh Duong \\ Diversification of metropolization trajectories in southern Vietnam. Exemple of \\ the competition between Ho Chi Minh City and Binh Duong.
}

Khac Minh Tran

\section{Introduction}

1 Depuis la mise en place des réformes du Đôi Moi en 1986, le développement du Vietnam est axé sur une industrialisation globalisée, accompagnée par la modernisation d'infrastructures techniques. Situées dans la région du Sud-Est du Vietnam ${ }^{1}$, les provinces de Hô Chi Minh Ville (HCMV) et de Binh Duong sont aujourd'hui devenues deux des pôles industriels majeurs, comptant 51 des 325 zones industrielles du pays en 2017 (Institut de planification du Sud, 2017; Département des zones économiques, 2017). En parallèle des nouvelles infrastructures industrielles, les périphéries urbaines de ces deux provinces connaissent aujourd'hui un développement immobilier rapide, se traduisant à la fois par une urbanisation autoproduite et par la création de centaines de complexes résidentiels investis par les promoteurs privés. La mise en concurrence entre HCMV et certaines provinces économiquement avancées, à savoir Dông Nai, Binh Duong et Ba Ria Vung Tau, ouvre de nouvelles perspectives de recherche, permettant de rendre compte des spécificités de la métropolisation au sud du Vietnam. Visant à décrypter la concurrence émergente entre HCMV et Binh Duong, ce travail s'inscrit dans la ligne directrice du dossier thématique «Hô Chi Minh Ville, terrain de jeux métropolitain(s) ». L'analyse portant sur le développement de ces deux provinces permet d'abord de caractériser certaines formes marquantes de la métropolisation, telles que l'industrialisation globalisée et les mégaprojets urbains. Ensuite, la recomposition des autorités provinciales et leurs liens avec certaines entreprises puissantes relèvent le caractère évolutif et complexe des jeux d'acteurs 
dans la production métropolitaine. Enfin, en centrant ses analyses sur les dynamiques interprovinciales, ce travail propose une lecture multiscalaire de la métropolisation de la région de HCMV.

Le développement de HCMV et de Binh Duong s'inscrit dans la trajectoire de croissance économique et urbaine de nombreuses métropoles d'Asie du Sud-Est, allant de la ville javanaise de Surabaya (Franck, 2010) aux capitales nationales de Bangkok (Deler, Kermel-Torrès, 2010) et de Kuala Lumpur (Bunnell et al., 2002 ; Bunnell, 2004). À l'heure de la métropolisation, un nouveau régime de production urbaine apparaît, qui introduit de nouvelles dynamiques spatiales, inspirées des logiques mondialisées et transgressant même les particularités locales (Goldblum, 2015). Dans le cas des villes vietnamiennes, la mise en place de ce régime se traduit par la coexistence de plusieurs modes d'urbanisation : autoconstruction liée aux initiatives populaires, mégaprojets érigés par l'État, projets immobiliers de promoteurs privés. En parallèle des capitales nationales et régionales, les villes secondaires, ainsi que certains arrière-pays des métropoles, émergent aujourd'hui comme de nouveaux pôles de développement. Les territoires émergents comme Binh Duong entrent aujourd'hui dans la première phase d'une métropolisation "en mode mineur", dont les spécificités se traduisent par un développement urbain et industriel à ampleur modérée et territorialement sélectif (Goldblum et al., 2012).

3 L'exemple de Binh Duong et de HCMV est révélateur de nouvelles tendances de réorientation de la stratégie d'industrialisation globalisée dans les pays émergents d'Asie. En parallèle du développement continuel des industries manufacturières exportatrices, ces deux provinces vietnamiennes s'engagent dans la course aux nouvelles technologies, qui participent aujourd'hui à transformer en profondeur l'appareil productif et à construire une nouvelle identité urbaine pour les métropoles émergentes d'Asie (Goldblum et al., 2012). Si les nouvelles technologies ne s'inscrivent que dans les stratégies urbano-industrielles des métropoles les plus compétitives, le développement des activités de promotion foncière et immobilière est accéléré et généralisé dans les villes de la région. Ces activités se développent rapidement, s'associant aux mégaprojets érigés par l'État dans l'objectif de déconcentrer les villescentres et de créer de nouvelles centralités métropolitaines (Henriot, 2013). L'urbanisme de projet conduit en conséquence à l'émergence d'un marché foncier et immobilier dynamique. Les promoteurs emploient de manière généralisée des stratégies de spéculation et de négociation informelle avec les autorités (Labbé et Musil, 2017 ; Musil et al., 2018), ce qui est révélateur de l'émergence d'un « capitalisme de connivence » (Beresford, 2008) dans le contexte autoritaire du Vietnam. Dans le cas de la région de HCMV, l'exemple du couple Binh Duong-Becamex a montré comment les autorités publiques tissent des liens étroits avec les entreprises, afin d'exercer leur influence sur le développement local. Créée en 1976 sous le nom de Bên $\mathrm{Cat}^{2}$ General Trading Company et entièrement possédée par le Comité populaire de Binh Duong, cette société et ses 28 filiales contrôlent les secteurs stratégiques de l'économie de cette province: immobilier résidentiel, zones industrielles et infrastructures routières. Comme les postes clés de Becamex sont en général attribués aux dirigeants locaux, le Comité populaire exerce un contrôle strict sur Becamex, ce qui permet d'éviter toute influence provenant de l'État central et des ministères.

4 Au-delà de son ambition métropolitaine, Binh Duong entretient toujours des liens d'interdépendance forts avec la métropole de HCMV. Inscrite dans la dernière vague 
d'industrialisation du Vietnam initiée à partir du milieu des années 2000, Binh Duong possède des ressources foncières abondantes, permettant un coût des terrains encore raisonnable. Cet atout permet à Binh Duong de renforcer l'attractivité de ses infrastructures industrielles, par rapport à celles de plus en plus saturées de HCMV. Pourtant, Binh Duong reste toujours dépendante des infrastructures routières et portuaires de HCMV, notamment pour l'acheminement et l'exportation de ses biens manufacturés. Avec une trajectoire de développement étroitement imbriquée dans celle de HCMV, Binh Duong cherche aujourd'hui à réinventer son modèle de développement, principalement dans l'objectif d'atténuer sa dépendance logistique envers la ville-province.

5 Si les métropoles nationales comme HCMV attirent une attention croissante des chercheurs, la province voisine de Binh Duong reste aujourd'hui un terrain peu connu de la recherche sur le Vietnam. Située au nord-est de la métropole de HCMV de plus de huit millions d'habitants (Hô Chi Minh City Statistical Office, 2017), la province de Binh Duong connait aujourd'hui un développement rapide. Cette province de deux millions d'habitants (Binh Duong Statistical Office, 2017) possède aujourd'hui 28 zones industrielles contre les 23 de la municipalité spéciale (Institut de planification du Sud, 2017). Le développement de Binh Duong s'appuie également sur la création de nombreuses infrastructures routières modernes, reliant ses pôles productifs aux complexes logistiques au sud de la région métropolitaine de HCMV (figure 1). En parallèle de son ambition de concurrencer la métropole de HCMV, qui s'affirme avec la création de plusieurs villes nouvelles et complexes urbano-industriels, cette province cherche à nouer des liens de complémentarité avec les territoires voisins. À l'heure de la métropolisation, comment les provinces de HCMV et de Binh Duong réinvententelles leurs stratégies urbano-industrielles, ce qui conduit au renforcement de la concurrence interprovinciale et à la redéfinition des dynamiques de construction régionale? 
Illustration 1 - Les provinces étudiées au sein de la région de HCMV

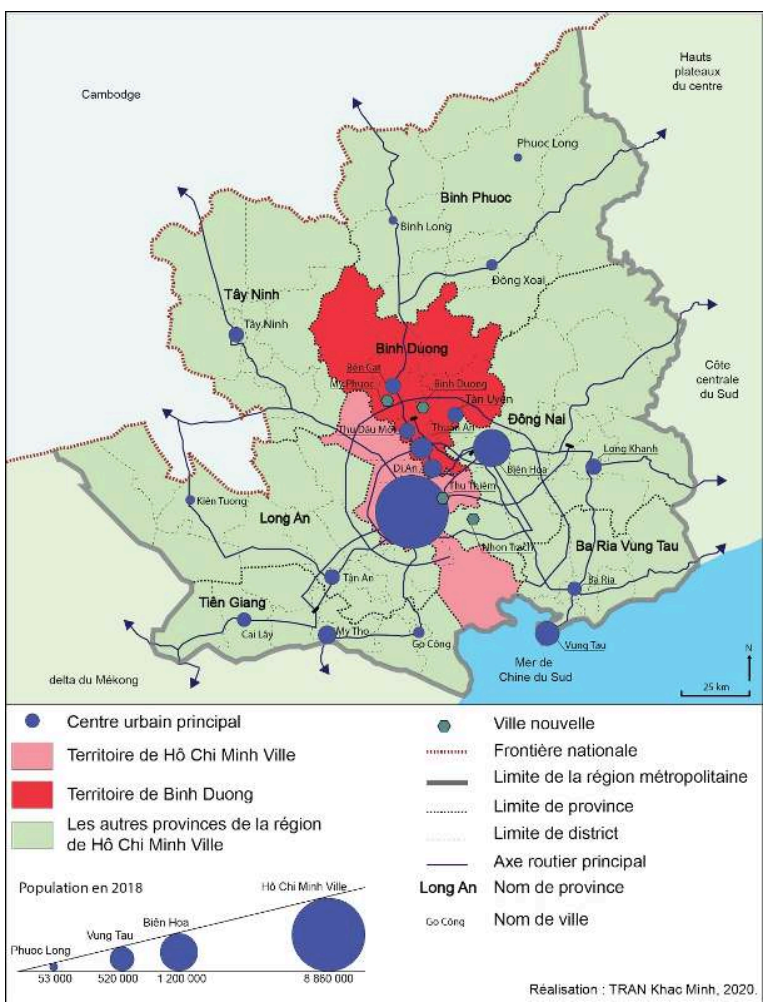

Sources: Ho Chi Minh City Statistical Office, 2017 ; Binh Duong Statistical Office, 2018 Binh Phuoc Statistical Office, 2018.

6 L'analyse et les données présentées ici sont les résultats d'un travail de recherche effectué entre 2015 et 2018 dans le cadre d'un doctorat de géographie (Tran, 2019). La méthodologie s'est appuyée sur trois démarches principales. D’abord, une série d'observations effectuée entre 2015 et 2019 a eu pour vocation de caractériser les formes spatiales des infrastructures d'envergures, des zones industrielles et des mégaprojets urbains. Ensuite, plusieurs entretiens semi-directifs ont été réalisés auprès des trois catégories d'acteurs suivantes : les autorités locales (province, sous-district, district), les promoteurs immobiliers et de zones industrielles, les chercheurs et consultants. Cette démarche a permis non seulement de mettre en évidence l'apparition de nouvelles logiques de concurrence, mais aussi de caractériser les liens d'alliance et de complémentarité existants entre ces acteurs. Enfin, la cartographie et l'analyse de données statistiques sont les deux outils les plus employés dans ce travail, dans le but d'appuyer les résultats obtenus lors des enquêtes de terrain. La structure de cet article est axée sur trois thématiques clés : l'industrialisation, l'urbanisme de projet et la construction régionale. Enfin, notre travail vise à mettre l'accent sur les rapports de force de plus en plus complexes, centrés autour des jeux de négociation et des conflits d'intérêts entre les territoires, ainsi qu'entre les autorités, les entreprises et la population locale. 


\section{Une industrialisation globalisée au cœur de la construction des territoires provinciaux}

7 La création de multiples zones industrielles à partir des années 1990 correspond aux différentes phases de la construction des territoires urbains des provinces étudiées. Comme l'industrialisation a contribué à transformer en profondeur l'économie locale, de nouveaux fronts d'urbanisation émergent et participent à la densification et à la transformation paysagère des périphéries métropolitaines.

8 Si HCMV a traversé toutes les phases de l'industrialisation du Vietnam à partir des réformes du Renouveau de 1987, la l'émergence de Binh Duong est marquée par une rapidité et une intensité remarquables, faisant de cette dernière une des provinces les plus industrialisées du pays. L'industrie représente en 2016 24,9\% du PIB de HCMV (Ho Chi Minh City Statistical office, 2016) et $63 \%$ de celui de Binh Duong (Binh Duong Statistical Office, 2016), ce qui permet d'affirmer le rôle dominant de l'industrialisation dans le développement de ces territoires. L'ampleur du développement de zones industrielles dans ces deux provinces se traduit par une croissance rapide des investissements directs étrangers. Dans le cas de HCMV, la métropole représente 12,5\% du montant (soit 42,52 milliards de dollars) et 31,9\% des projets d'investissements directs étrangers au Vietnam entre 1988 et 2016 (Ho Chi Minh City Statistical Office, 2016). Jusqu'en 2016, 5,41 milliards de dollars d'investissements directs étrangers ont été versés dans les zones industrielles et les zones franches de la ville-province, auxquels s'ajoutent plusieurs milliards de dôngs d'investissements privés nationaux. Malgré cela, les établissements à investissements directs étrangers ne représentent que $35 \%$ de la production industrielle brute de HCMV, contre les $51 \%$ des entreprises nationales (Hô Chi Minh City Statistical Office, 2016).

Quant à la province émergente de Binh Duong, son secteur manufacturier à investissement étranger joue un rôle encore plus marquant qu'à HCMV. Jusqu'en 2016, les entreprises étrangères ont investi plus de 19 milliards de dollars dans le secteur manufacturier de la province. En 2016, le secteur manufacturier à investissements directs étrangers représente $67,5 \%$ de la production industrielle de Binh Duong (Binh Duong Statistical office, 2016). Le rôle de l'industrialisation dans l'intégration internationale de Binh Duong et de HCMV se traduit enfin par la croissance rapide et stable des exportations. Dans le cas de Binh Duong, les exportations réalisées par les industries légères et les filières artisanales sont passées de 8,1 à 19,2 milliards de dollars entre 2011 et 2016 (Binh Duong Statistical Office, 2016). Générant 15,3 milliards de dollars en 2005, les exportations réalisées par HCMV ont atteint 29,3 milliards de dollars en 2016, soit une croissance de $195 \%$ en onze ans (Ho Chi Minh City Statistical office, 2016).

Malgré des résultats encourageants, l'industrialisation de HCMV et de Binh Duong est marquée par une dépendance croissante envers les importations de matières premières et d'équipements de production. En 2016, Binh Duong a par exemple importé 20,4 milliards de dollars de matières premières et d'équipements de production. Le secteur du textile et du cuir, considéré comme un fer de lance de l'industrie de Binh Duong, a importé en 2016 plus de 2,4 milliards de matières premières (Binh Duong Statistical Office, 2016). En parallèle des fragilités liées aux fluctuations du marché mondial de matières premières, le poids des activités d'assemblage reste important dans la structure de l'industrie de ces deux provinces. Le secteur manufacturier de Binh Duong 
et de HCMV retrouve son attractivité encore dans les sources abondantes de maind'œuvre bon marché, qui est aujourd'hui de plus en plus concurrencée par les pays voisins comme le Cambodge et le Laos.

11 L'industrialisation de HCMV et de Binh Duong est également caractérisée par des tendances spatiales spécifiques, conduisant à la diversification de modes de développement des réseaux provinciaux de zones industrielles. Deux logiques ont été identifiées. Si les zones industrielles de HCMV se sont développées, principalement durant les années 1990 et 2000, selon une forme concentrique autour de la ville-centre, celles de Binh Duong ont été créées en formant des corridors nord-sud, le long des axes routiers majeurs et desservant les pôles urbains principaux.

Les ceintures de zones industrielles de HCMV se sont développées au nord, à l'ouest et à l'est de la métropole (illsutrations 2 et 3 ). Si les zones industrielles de la ceinture orientale se sont développées en synergie avec celles de Binh Duong et de Dông Nai, les infrastructures créées au nord correspondent à l'intention de la municipalité de transformer le district rural de $\mathrm{Cu} \mathrm{Chi}$ en une ville-satellite pour déconcentrer la villecentre (Institut de planification du Sud, 2017). Quant à la ceinture de l'ouest, l'abondance de ressources foncières est l'un des éléments justifiant la création de nouvelles zones industrielles depuis les années 2000, principalement dans les secteurs limitrophes des provinces de Tiên Giang et de Long An. Selon les données présentées dans le schéma directeur de la région de HCMV de 2017, la ville-province tend à reconvertir quatre sites industriels ${ }^{3}$ en zones industrielles d'ici 2030. En parallèle de cette stratégie, huit zones industrielles font l'objet de projets d'élargissement, ce qui permettra de générer 2800 ha destinés au secteur manufacturier d'ici 2020 (Institut de planification du Sud, 2017). Malgré ces politiques visant à élargir le secteur manufacturier local, l'industrie ne semble plus le fer de lance de l'économie de la métropole. Son secteur secondaire représente en part relative $48,1 \%$ du PIB de la villeprovince en 2005, 44,1\% en 2008, 37,9\% en 2011, avant de chuter à $24,9 \%$ en 2016 (Ho Chi Minh City Statistical Office, 2011, 2015, 2016). Dans les districts de l'est (districts 2, 9 et de Thu Duc), dont le développement durant les années 1990 et 2000 s'est appuyé sur les zones industrielles, la promotion foncière et immobilière est aujourd'hui un nouveau moteur de l'économie locale, remplaçant le secteur manufacturier. Si les nouvelles stratégies de développement de HCMV contribuent à atténuer le rôle de l'industrie au profit du tertiaire et du secteur de l'immobilier, Binh Duong élabore aujourd'hui de nouvelles politiques visant à renforcer le rôle du secteur secondaire. 
Illustration 2 - Les infrastructures industrielles et logistiques de HCMV

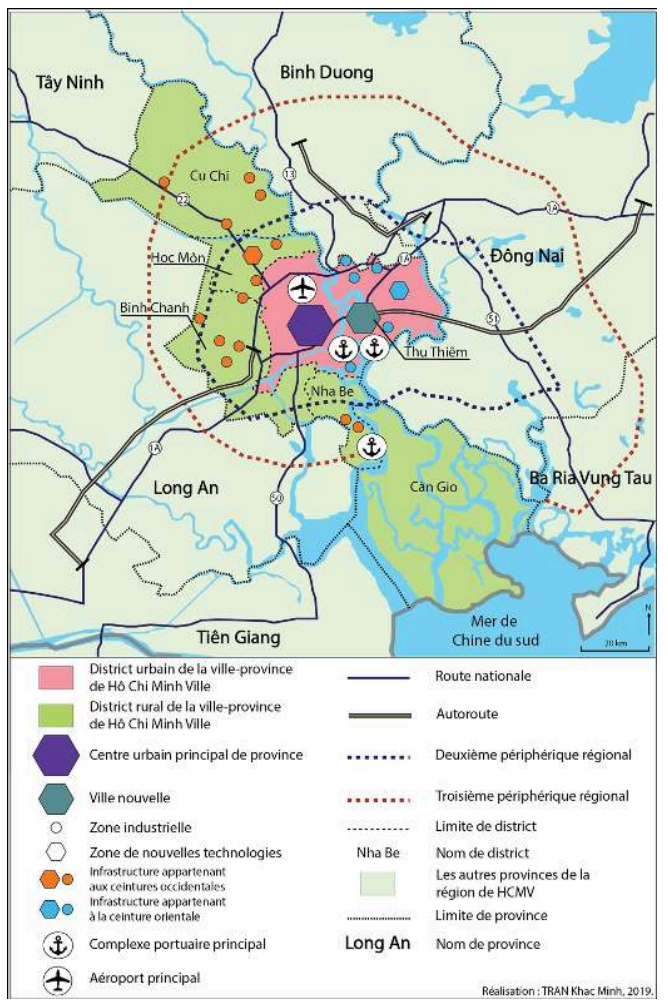

Source : Institut de planification du Sud, 2017.

Illustration 3 - La zone industrialo-portuaire de Cat Lai dans le district 2 de HCMV

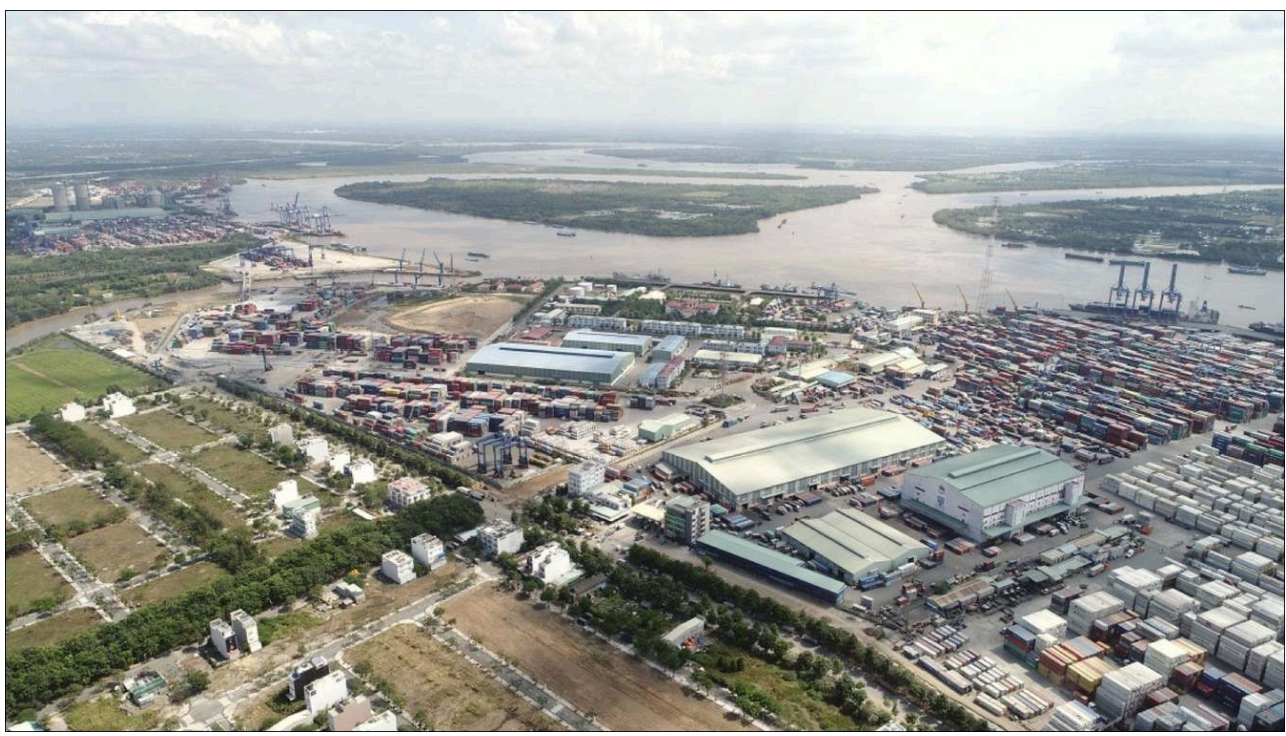

Source : SomoGroup, 2018.

$13 \mathrm{Au}$ contraire de la forme concentrique du réseau d'infrastructures industrielles de HCMV, les pôles manufacturiers de Binh Duong sont reliés par certains axes routiers principaux (la route nationale $n^{\circ} 13$ et l'autoroute My Phuoc-Tân Van) en formant un corridor nord/sud (illustration 4). À partir des zones industrielles développées dans les districts du sud (Di An et Thuân An) dans les années 1990, les territoires du centre 
(Thu Dâu Môt, Tân Uyên et Bên Cat) émergent depuis les années 2000, comme nouveaux pôles manufacturiers de la province. En plaçant les territoires du centre et du nord au centre de son appareil productif, Binh Duong cherche à échapper aux influences de HCMV, en s'affirmant comme l'unique pôle manufacturier au nord de la région du HCMV.

Comme cette répartition territoriale des zones industrielles est révélatrice de la volonté de la province de modeler les dynamiques locales de développement, Binh Duong a réussi à devancer HCMV dans le domaine manufacturier, au moins sur deux points. Premièrement, la surface destinée aux zones industrielles ( 9400 ha pour 28 zones industrielles) de Binh Duong dépasse aujourd'hui largement celle de HCMV (3900 ha pour 23 zones industrielles) (Institut de planification du Sud, 2017). La surface moyenne des zones industrielles de Binh Duong ( $340 \mathrm{ha}$ ) est deux fois supérieure à celle de HCMV (170 ha), ce qui permet à cette première d'accueillir des usines de taille importante.

Deuxièmement, la province de Binh Duong a élaboré un modèle unique de développement, qui permet d'associer l'industrialisation au secteur immobilier. Les zones industrielles de HCMV se développent dans les secteurs périphériques de plus en plus éloignés de la ville-centre, ce qui permet de réserver les terrains constructibles dans les districts péricentraux à la promotion immobilière. À l'opposé de cette stratégie, Binh Duong encourage la construction de zones industrielles à proximité des pôles urbains, dans l'objectif de créer des liens de complémentarité entre l'industrie et le secteur immobilier (illustration 5). Initialement développé par Becamex, le modèle de «zone urbano-industrielle» consiste à favoriser l'implantation de projets immobiliers dans les espaces limitrophes des zones industrielles. Les cinq zones urbano-industrielles de Binh Duong, dont la taille peut varier de 700 (la zone VSIPViêt Sing dans le district de Thuân An) à plus de 6000 ha (la ville nouvelle de My Phuoc dans le district de Bên Cat), sont entièrement développées par Becamex, ce qui met en évidence le rôle déterminant de cette société publique dans l'économie locale. 
Illustration 4 - Les infrastructures industrielles et logistiques de Binh Duong

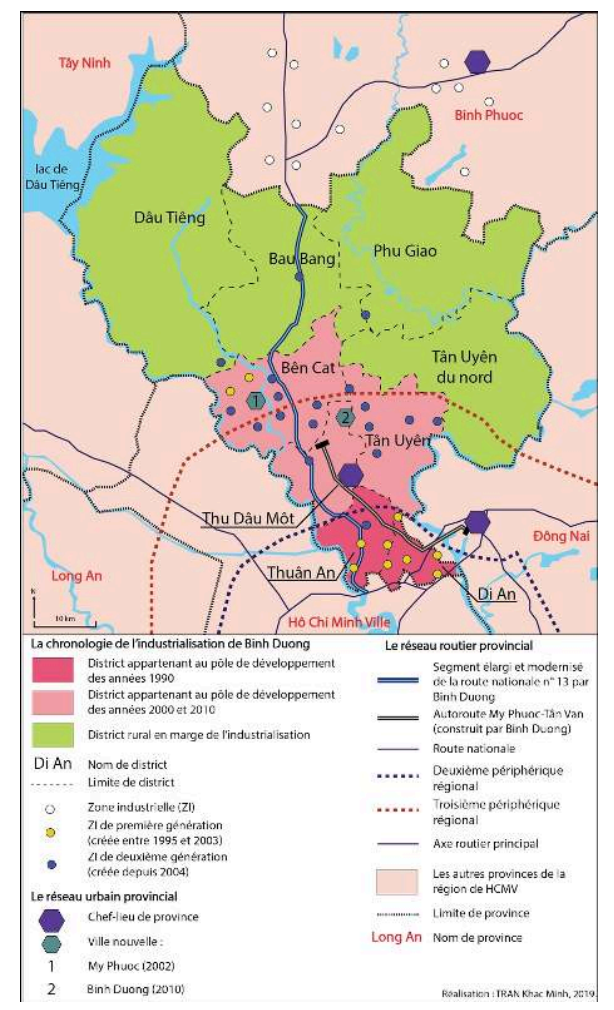

Source : Institut de planification du Sud, 2017.

16 Au-delà des stratégies différentes, HCMV et Binh Duong développent aujourd'hui de nouveaux secteurs clés: les nouvelles technologies, le tertiaire et l'immobilier. Si l'ambition technopolitaine de ces provinces se traduit par la création de trois zones de nouvelles technologies (deux à HCMV et une à Binh Duong), le développement rapide du secteur de l'immobilier a conduit à l'émergence de nouveaux fronts d'urbanisation, stimulés par un urbanisme de projet dynamique. 
Illustration 5a - Les zones industrielles de Binh Duong : zone tertiaire et de nouvelles technologies Mappletree

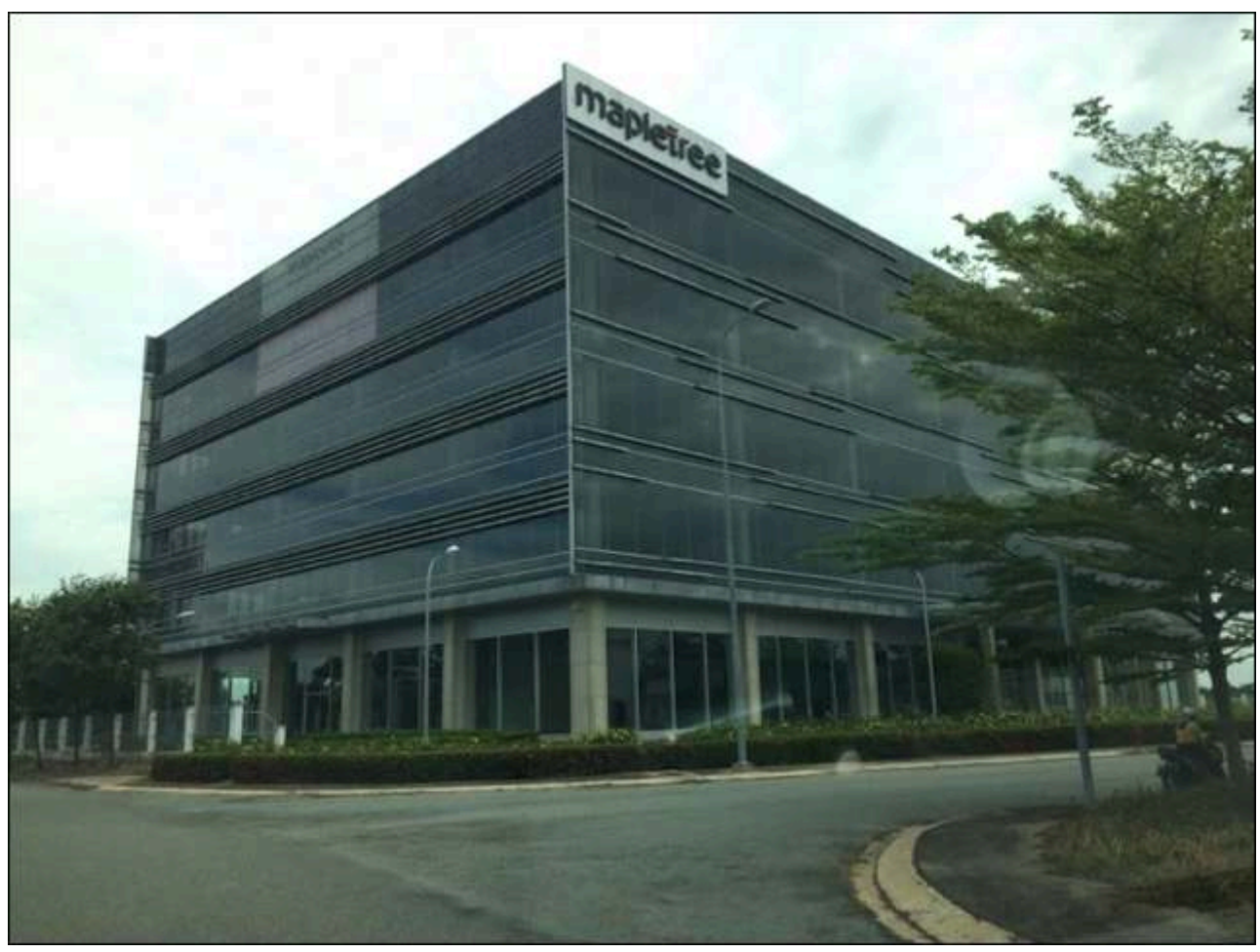

Auteur: Khac Minh Tran, 2015.

Illustration 5b - Les zones industrielles de Binh Duong : La zone industrielle de VSIP I

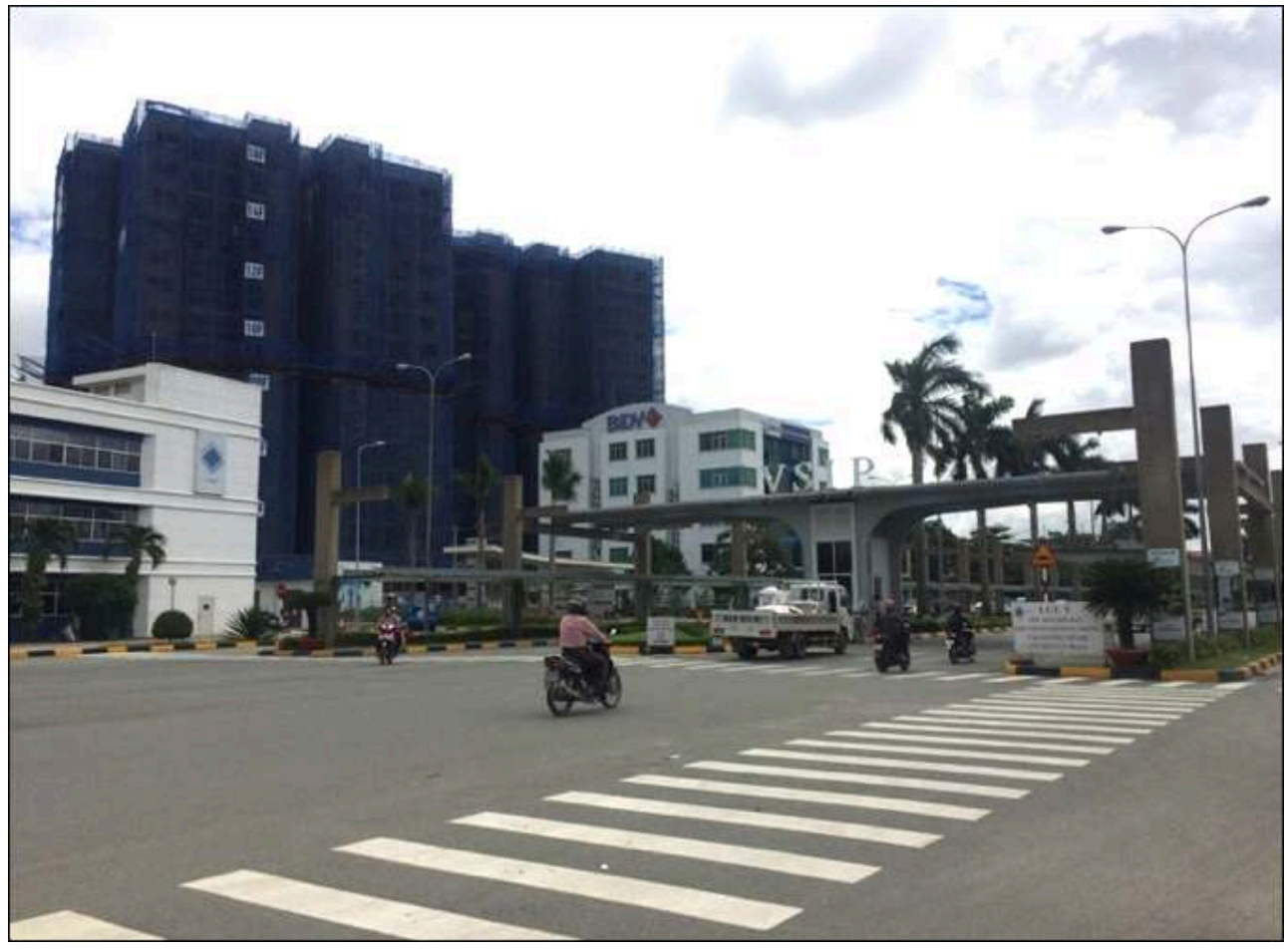

Auteur: Khac Minh Tran, 2018. 


\section{Un urbanisme de projet dynamique comme moteur de la métropolisation}

17 En parallèle de l'urbanisation autoproduite menée par la population, la promotion foncière et immobilière a permis non seulement l'expansion des territoires urbains de HCMV et de Binh Duong, mais aussi d'introduire de nouveaux modes d'organisation socio-spatiale. Dans le contexte actuel du développement urbain rapide, le rôle des autorités et de certains groupes d'acteurs s'affirme, à travers l'emploi de nouveaux outils de planification, de négociation et de financement. HCMV et Binh Duong ont mis en place des stratégies d'urbanisation différentes, qui permettent de modeler leurs dynamiques spatiales de développement et de renforcer leur compétitivité.

La municipalité de HCMV ne joue aujourd'hui qu'un rôle régulateur dans son secteur immobilier, en adoptant des modes de coordination de moins en moins contraignants envers les promoteurs. En conséquence de cette politique, la configuration du marché immobilier local est segmentée entre les firmes 50 送 qui investissent dans certains projets de taille importante 㡠 et une multitude de promoteurs nationaux dotés d'une marge de manœuvre considérablement moins conséquente (Nguyen et al., 2014). À l'opposé de HCMV, le secteur immobilier de Binh Duong est principalement contrôlé par Becamex, qui exerce comme le bras droit du Comité populaire.

19 En 2014, $36 \%$ des projets immobiliers de Binh Duong sont développés par des sociétés publiques, majoritairement par Becamex. La superficie des projets pris en charge par ces dernières dépasse considérablement celle des zones urbaines nouvelles développées par les entreprises privées (6 400 contre 2320 ha) (Centre de recherche sur l'habitat et sur l'immobilier de Binh Duong, 2014). La surface moyenne d'une zone urbaine nouvelle développée par les sociétés publiques s'élève en conséquence à 136 ha, contre 31 pour les projets privés. Enfin, $61 \%$ des logements issus de zones urbaines nouvelles sont produits par les sociétés publiques, contre $35 \%$ par les entreprises privées. Ces constats permettent de mettre en évidence le rôle prédominant de Becamex et de ses filiales dans le développement du marché immobilier de la province. Si HCMV attire à tout prix les investisseurs étrangers et les firmes nationales, comme Vingroup et Novaland, même au prix de l'affaiblissement de son pouvoir régulateur, Binh Duong place son bras droit Becamex au centre du marché immobilier. Cette stratégie a permis à la fois d'écarter des concurrents venus de l'extérieur et de modeler le développement urbain selon les stratégies prédéfinies.

Les cadres conceptuels des stratégies urbaines de ces deux provinces se distinguent fondamentalement. Si Binh Duong cherche à associer l'industrie à la promotion immobilière, HCMV concentre ses efforts dans un développement urbain de plus en plus déconnecté de l'industrialisation. La municipalité favorise aujourd'hui le développement de son parc résidentiel et de certains mégaprojets urbains, dans l'intention de développer HCMV selon l'image des métropoles mondiales d'Asie. Dans cette optique, le développement urbain s'appuie sur la réhabilitation du centre-ville, avec la construction de plusieurs tours de bureaux. La verticalisation de l'hypercentre de HCMV provoque pourtant plusieurs problèmes. Face à la congestion urbaine et aux risques croissants d'inondation, liée à l'artificialisation du sol, le Comité populaire de HCMV a dû mettre un terme en 2018 à la construction d'immeubles de grande hauteur dans les districts du centre-ville (Résolution 10/NQ-HĐND/2018). Dans ce contexte, la construction de la vitrine métropolitaine doit reposer désormais sur le développement 
des secteurs péricentraux et périphériques. Les 104 projets immobiliers développés dans le centre-ville, étendus sur 68 ha, ne représentent que 8,5\% des zones urbaines nouvelles et $1 \%$ de la surface destinée à la promotion immobilière de HCMV (Pham et al., 2017). Les districts 2 et 9 de Binh Thanh et de Thu Duc constituent aujourd'hui un pôle urbain majeur, en polarisant un tiers des projets immobiliers et plus de $40 \%$ de la surface destinée à la promotion immobilière. Appartenant aux ceintures péricentrales et périphériques de l'est, ces districts possèdent également certains des mégaprojets emblématiques de HCMV : la ville nouvelle de Thu Thiêm (illustration 6), le complexe résidentiel Vinhomes Central Park (figure 7) et le projet en construction VinCity du district 9.

21 Les mégaprojets développés dans les espaces péricentraux et périphériques de HCMV sont communément déconnectés des zones industrielles de la ville-province. À prédominance résidentielle, la ville nouvelle de Thu Thiêm (district 2) et le complexe Vinhomes Central Park (district de Binh Thanh) offrent également des conditions propices aux activités tertiaires. Au cœur de Thu Thiêm, Dai Quang Minh, l'un des promoteurs majeurs de la ville nouvelle, a construit une tour de bureaux, qui fonctionne aujourd'hui comme le centre de commandement de cette firme immobilière. Dans le complexe Vinhomes Central Park, VinGroup, qui est aujourd'hui le plus grand promoteur immobilier du Vietnam, a construit un hôpital international, des établissements éducationnels, et notamment la tour Landmark 81 (81 étages, $461 \mathrm{~m}$ de hauteur). Avec la construction ex nihilo de Thu Thiêm, Landmark 81 et Vinhomes Central Park sont aujourd'hui devenus le symbole de la verticalisation et la modernisation de HCMV (illustration 7).

Illustration 6 - La ville nouvelle de Thu Thiêm (district 2), un nouveau chantier métropolitain à l'est de HCMV

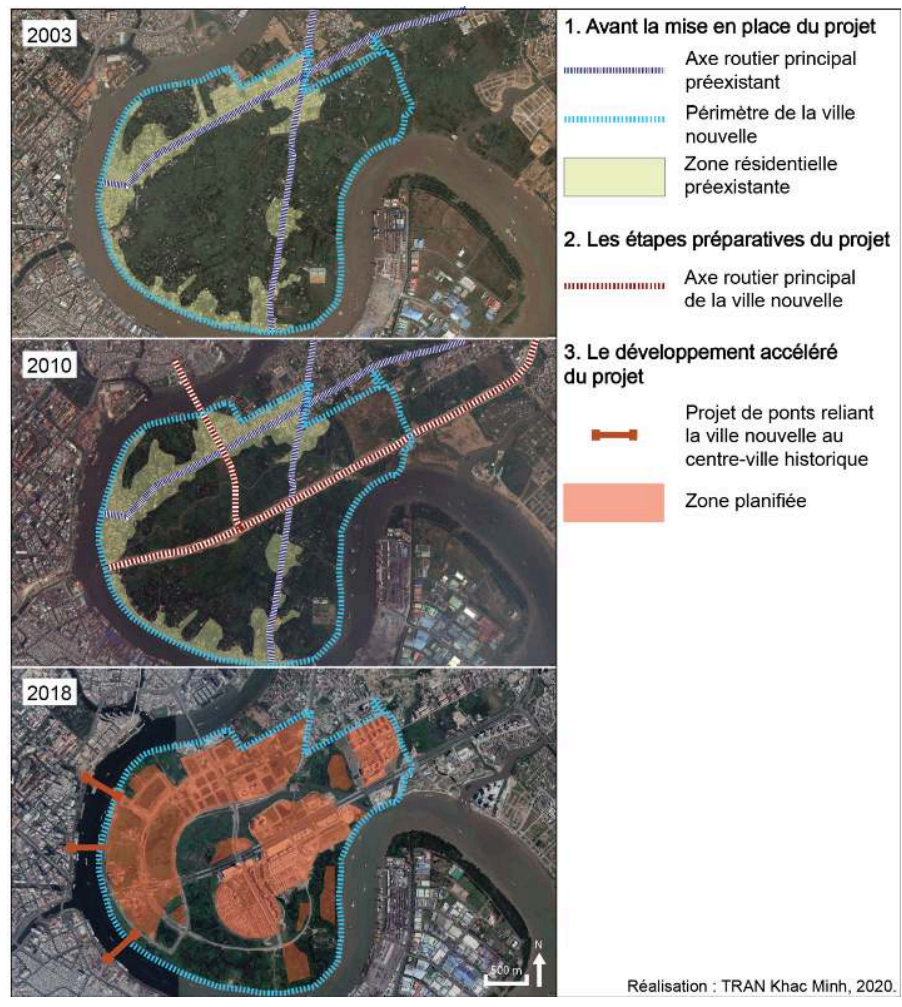

Source : Khac Minh Tran, 2019 
Illustration 7 - Le complexe résidentiel Vinhomes Central Park et la tour Landmark 81 (district de Binh Thanh)

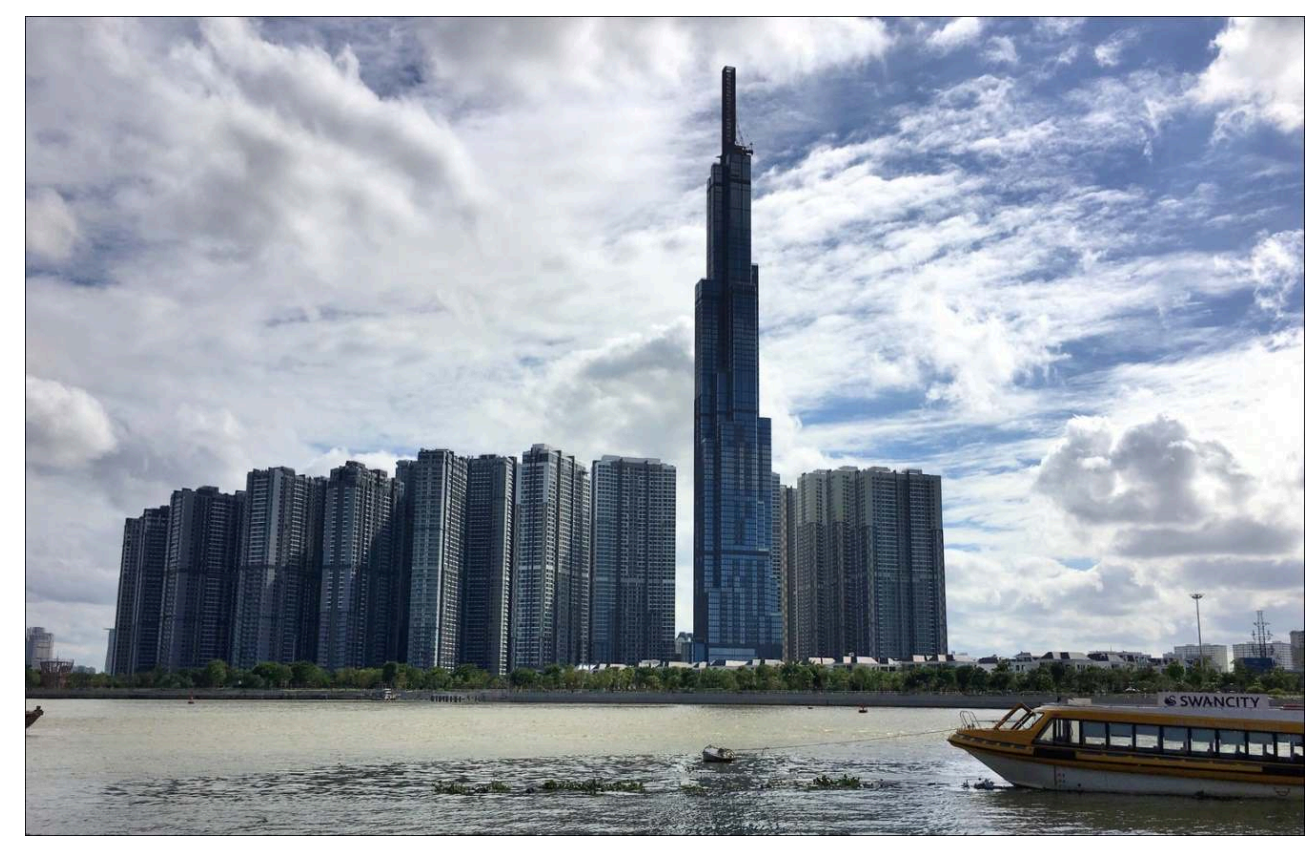

Auteur : Khac Minh Tran, 2018.

22 Au contraire de HCMV, Binh Duong concentre ses efforts dans la construction d'une vitrine métropolitaine associant l'industrialisation à l'urbanisme de projet (illustration 8). À l'instar du modèle de zone urbano-industrielle développé par Becamex, Binh Duong appuie son développement sur trois mégaprojets. La ville nouvelle de My Phuoc (6 200 ha, district de Bên Cat) et le complexe urbain industrialotertiaire de Binh Duong (4 200 ha, étendu sur le territoire de Thu Dâu Môt, de Bên Cat et de Tân Uyên) polarisent par exemple 10 des 28 zones industrielles de Binh Duong. À partir des années 2010, le développement de la ville nouvelle de Binh Duong (1120 ha), comme une partie intégrante du complexe urbain industrialo-tertiaire de Binh Duong, représente une étape décisive de la métropolisation de cette province. 
Illustration 8. Les mégaprojets urbains de la province de Binh Duong.

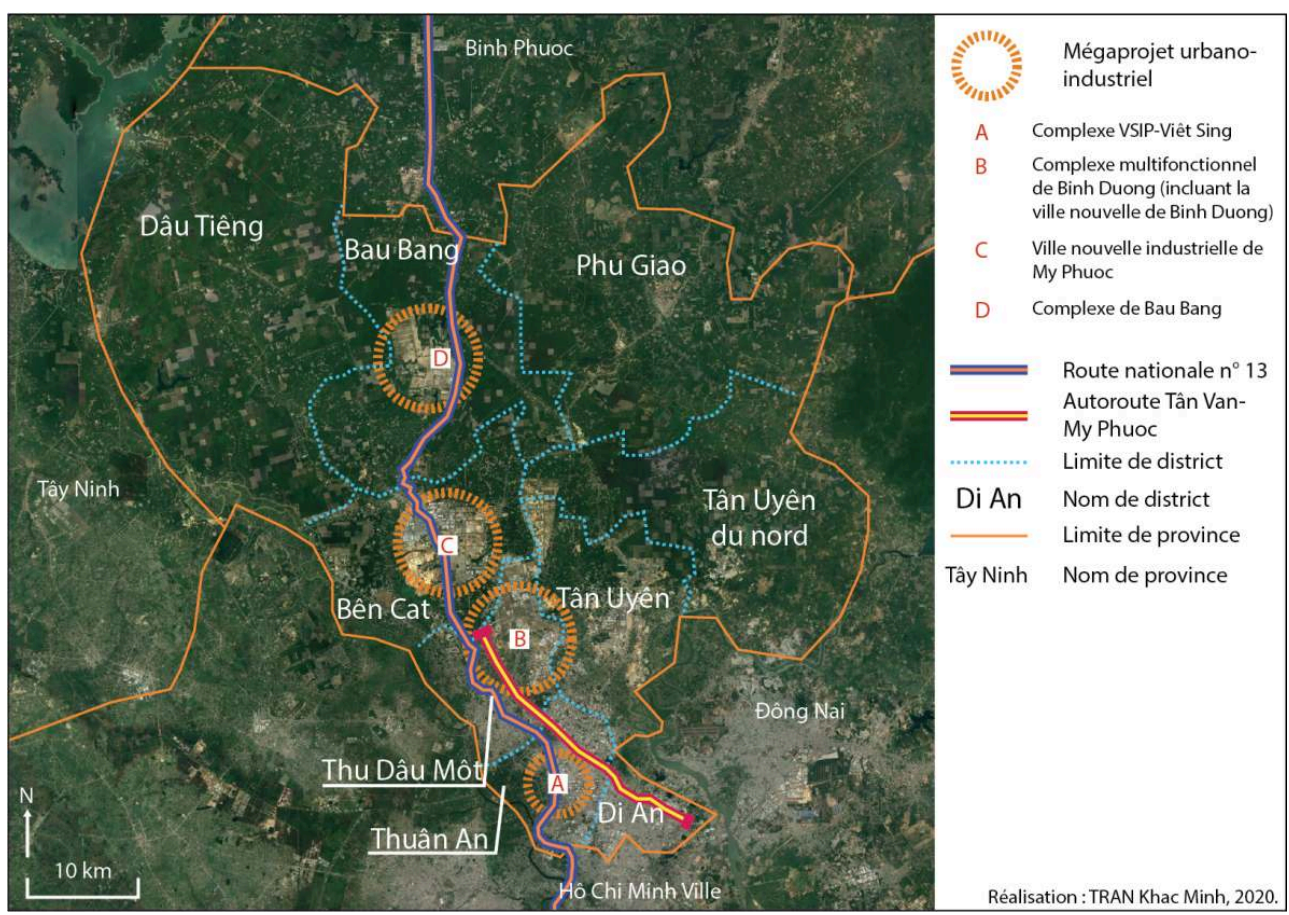

Auteur : Khac Minh Tran, 2019.

Exclusivement réservée aux nouvelles technologies, au tertiaire supérieur et à l'immobilier résidentiel haut de gamme, la ville nouvelle de Binh Duong a vocation de devenir le centre de commandement principal de la province (illustration 9). En parallèle de la construction d'un nouveau centre politico-administratif, cette ville nouvelle accueille également une zone de nouvelles technologies de 75 ha (investie et gérée par l'entreprise singapourienne MapleTree), le siège social de Becamex et des universités internationales. Par rapport au projet à prédominance résidentielle de Thu Thiêm, la ville nouvelle de Binh Duong est un exemple emblématique montrant l'ambition du Comité populaire de transformer Binh Duong en un pôle métropolitain tourné vers les nouvelles technologies et le tertiaire supérieur.

En parallèle de sa spécificité sectorielle, le lien de synergie entre la ville nouvelle de Binh Duong et le chef-lieu de Thu Dâu Môt joue également un rôle fondamental dans l'émergence des territoires du centre de cette province. La modernisation du réseau intraurbain de Thu Dâu Môt depuis de la fin des années 2000 a permis d'assurer une liaison rapide entre ces deux localités. Thu Dâu Môt se trouve également au cœur d'un programme provincial élaboré par Becamex visant à développer des «villes intelligentes » sur le territoire de Binh Duong, qui s'articulent autour des nouvelles technologies, du tertiaire supérieur, de la recherche scientifique et de l'innovation. Le développement de la ville nouvelle de Binh Duong s'inscrit en effet dans une double dynamique: une extension urbaine par le dédoublement du centre-ville de Thu Dâu Môt, accompagnée par la mise en place du concept de « ville intelligente ».

Si Becamex a réussi à conserver son monopole dans le développement du marché immobilier de Binh Duong face aux concurrents nationaux, le bras droit du Comité populaire se lance dès les années 2000 dans plusieurs programmes de coopération internationale. La ville nouvelle de Binh Duong émerge comme le symbole de la 
diversification des partenaires de Becamex. En parallèle de la zone de nouvelles technologies MapleTree, qui a réussi à attirer plus de 400 millions de dollars (Ville nouvelle de Binh Duong, 2019), la joint-venture vietnamo-japonaise Becamex-Tokyu est aujourd'hui devenue le plus grand promoteur immobilier de la ville nouvelle. L'emblématique projet Tokyu Binh Duong Garden City, étendu sur 110 ha, a absorbé plus de 1,2 milliard de dollars d'investissements (Becamex, 2019). Des condominiums certifiés de "qualité japonaise » aux futurs centres commerciaux et centres d'affaires, le Comité populaire de Binh Duong tend à faire de cette ville nouvelle un pôle métropolitain au nord de la région, capable d'entrer en concurrence avec le centre d'affaires de HCMV.

Au-delà du développement continuel des marchés immobiliers locaux, HCMV et Binh Duong appuient leur développement urbain sur des mégaprojets métropolitains. Le développement du secteur immobilier, des nouvelles technologies et du tertiaire supérieur, dans le cadre des zones urbaines nouvelles, s'inscrit dans une stratégie visant à favoriser l'intégration mondiale de ces deux provinces les plus développées de la région de HCMV. La mise en complémentarité et en concurrence entre les pôles métropolitains conduit aujourd'hui à la formation de nouveaux corridors intrarégionaux, reliant les territoires productifs des provinces économiquement avancées de la région de HCMV.

Illustration 9 - Les projets de coopération entre Becamex et les acteurs étrangers comme moteurs du développement de la ville nouvelle de Binh Duong

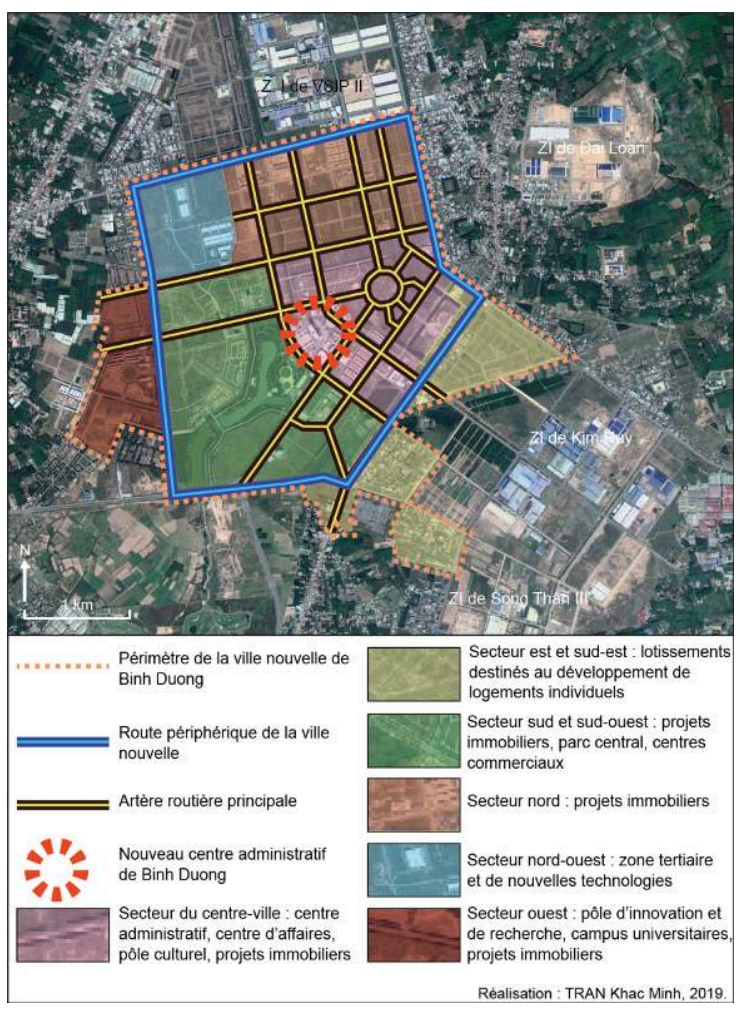

Auteur : Khac Minh Tran, 2019. 


\section{Les corridors interprovinciaux : entre complémentarité, concurrence et contournement} conduit à l'émergence de nouveaux pôles de développement au sein des territoires provinciaux de HCMV et de Binh Duong. Si la mise en relation entre ces pôles au sein des territoires provinciaux s'accélère sous l'effet de la modernisation d'infrastructures techniques, de nouvelles dynamiques de complémentarité avec les autres provinces voisines de la région de HCMV voient le jour. Alors que HCMV cherche à conserver son pouvoir hégémonique, construit autour de sa puissance économique et de ses infrastructures performantes, Binh Duong tend aujourd'hui à développer de nouveaux projets de coopération logistico-industrielle avec les provinces voisines. L'émergence de ces nouvelles logiques de coopération, inhérentes à la concurrence entre HCMV et Binh Duong, redéfinit et complexifie les dynamiques de construction de la plus grande région métropolitaine du Vietnam.

Si les zones industrielles situées à l'est de HCMV, directement relié à celles du sud de Binh Duong, ont joué un rôle fondamental dans le développement des années 1990, les dynamiques d'industrialisation de la ville-province se concentrent aujourd'hui dans sa partie occidentale. Cette partie, regroupant 17 des 23 zones industrielles de HCMV, se développe en continuité avec celles des provinces voisines de Long An et de Tiên Giang. Cette ceinture industrielle interprovinciale polarise aujourd'hui 31 zones industrielles et se dote de nombreux potentiels au profit de l'industrialisation dans le futur: ressources foncières importantes, réseau routier dense (autoroute HCMV-Trung Luong, route nationale $\mathrm{n}^{\circ} 1$ et les périphériques régionaux) et sources abondantes de maind'œuvre. Le renforcement des dynamiques de développement des districts occidentaux de HCMV, qui encourage directement la mise en complémentarité avec Long An et Tiên Giang, permet de concurrencer Binh Duong et ses infrastructures industrielles. 


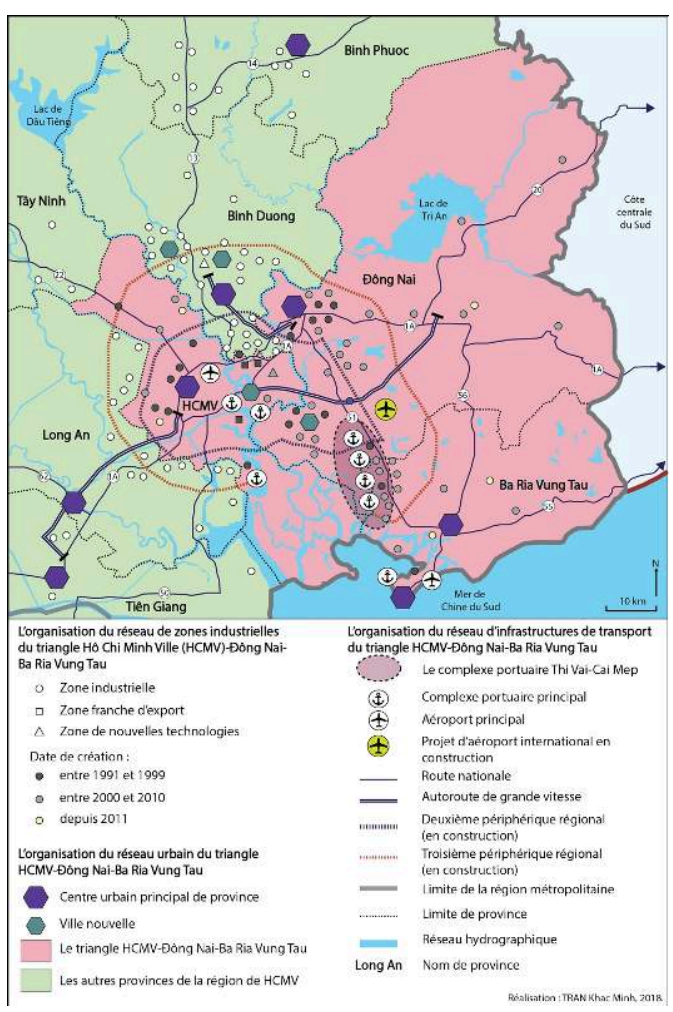

Source : Institut de planification du Sud, 2017.

Dans l'objectif de maintenir son rôle dominant au sein de la région, HCMV renforce les liens de complémentarité avec les provinces de Dông Nai et Ba Ria Vung Tau (figure 10). Le développement du triangle HCMV-Dông Nai-Ba Ria Vung Tau, amorcé dès les années 1990, est aujourd'hui renforcé par la construction de nouvelles infrastructures techniques. Premièrement, la construction de l'autoroute CT01 $(55 \mathrm{~km}$, mise en service en 2015) a permis une liaison rapide entre la ville-centre de HCMV, le sud de Dông Nai et le nord de Ba Ria Vung Tau. Deuxièmement, le développement du complexe portuaire Thi Vai-Cai Mep ( $48 \mathrm{ha}$, mise en service en 2005) dans la province de Ba Ria Vung Tau s'inscrit dans une stratégie de renforcement des liens de complémentarité logistique avec HCMV. Prenant en charge $85 \%$ du volume des exportations par conteneurs réalisées par les provinces du Sud du Vietnam (VOV5, 2014), ce complexe portuaire est facilement relié aux zones industrielles de HCMV et de Dông Nai par l'autoroute $\mathrm{CT} 01$ et la route nationale $\mathrm{n}^{\circ} 51$. La délocalisation des infrastructures portuaires vers le sud de la région représente un nouveau défi pour l'industrialisation de Binh Duong. Si la distance importante entre les zones industrielles de Binh Duong et les ports est une faiblesse inhérente à la géographie de cette province, la saturation aggravée des axes routiers principaux force cette dernière à développer d'autres alternatives logistiques pour protéger sa compétitivité.

L'autoroute My Phuoc-Tân Van (30 km, en construction depuis 2009), reliant les pôles urbano-industriels du centre de Binh Duong à la province de Dông Nai, permet aujourd'hui de contourner les axes routiers de plus en plus saturés de HCMV (illustration 11). La mise en service de cette autoroute contribue à créer de nouveaux liens de complémentarité entre Binh Duong et Dông Nai, tout en atténuant la dépendance logistique de cette première envers HCMV. En parallèle du corridor nord- 
sud formé avec Đông Nai, Binh Duong se lance aujourd'hui dans un programme de coopération urbano-industrielle avec la province frontalière de Binh Phuoc. Pris en charge par Becamex, ce programme consiste à construire un grand complexe urbanoindustriel à Binh Phuoc. Créé en 2005, ce projet de plus de 4500 ha fait partie d'un nouveau front d'industrialisation, en continuité avec les zones industrielles de Binh Duong (illustration 12). Au-delà des logiques de coopération bilatérale, cette initiative peut contribuer à créer de nouvelles dynamiques de développement transfrontalier avec le Cambodge dans le futur. Au gré de l'avancée de ce front de développement, un corridor nord-sud, regroupant plus de 40 zones industrielles et 3 millions d'habitants (Vietnam Statistical Office, 2016), se façonne et sera susceptible d'entrer en concurrence avec le pôle métropolitain formé par HCMV et les territoires voisins. Sous l'effet de la mise en concurrence entre HCMV et Binh Duong, de nouvelles dynamiques transprovinciales, orientées vers les espaces longtemps en marge, émergent et complexifient la configuration régionale, qui est jusque-là marquée par le rôle prédominant d'un développement tourné vers le littoral.

Illustration 11 - L'organisation du réseau urbano-industriel de l'axe Binh Duong-Đông Nai

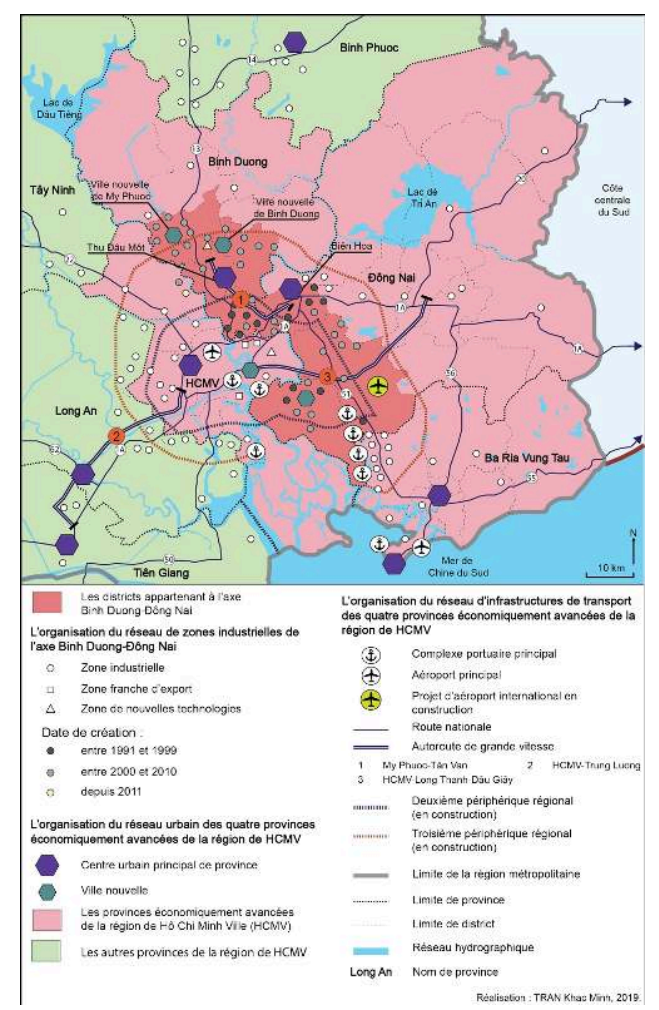

Source : Institut de planification du Sud, 2017. 


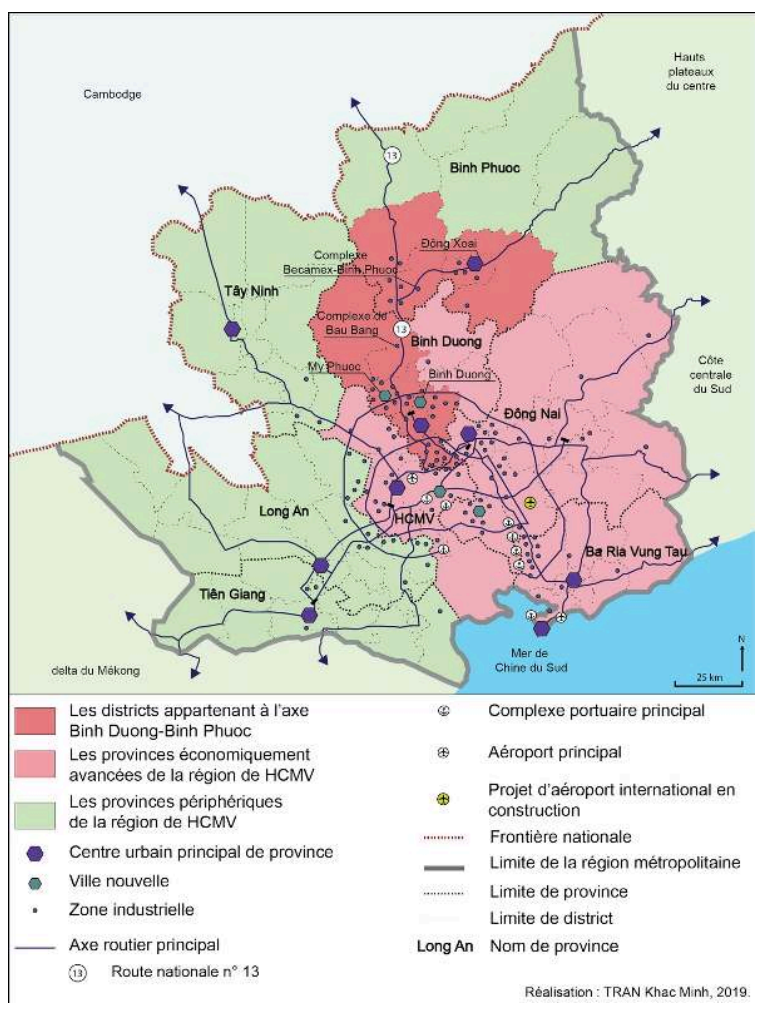

Source : Institut de planification du Sud, 2017.

31 L'émergence contemporaine de HCMV et de Binh Duong en tant que pôles métropolitains majeurs met en évidence le rôle fondamental de l'industrialisation et de l'urbanisme de projet dans la transformation du système urbano-économique des villes vietnamiennes. HCMV et Binh Duong sont avant tout un observatoire privilégié pour comprendre l'intégration du Vietnam dans la mondialisation. Au-delà de la création généralisée de zones industrielles et le développement accéléré de l'immobilier, les deux provinces ont mis en place dès les années 1990 des stratégies de métropolisation différentes, dans l'objectif de renforcer leur compétitivité. Si HCMV ne place plus l'industrie au cœur de son économie, au profit du tertiaire et de l'immobilier, le développement rapide de Binh Duong s'appuie sur la mise en complémentarité entre l'industrialisation et l'urbanisme de projet, sous l'égide de du couple Comité populaireBecamex. Dans l'objectif de renforcer leur capacité concurrentielle, HCMV et Binh Duong tissent de nouveaux liens de complémentarité avec les provinces voisines, principalement dans le cadre de projets d'infrastructures logistiques communs et de programmes de coopération industrielle.

Sous l'effet de la concurrence interprovinciale, les trajectoires de développement local se diversifient. L'exemple de la mise en concurrence entre Binh Duong et HCMV a permis de montrer la capacité d'adaptation remarquable des autorités locales au contexte actuel de montée en puissance des entreprises privées. Les stratégies adoptées par les provinces sont multiples, allant de la création de liens d'alliance étroits avec les firmes privées au renforcement de leur pouvoir économique par le biais d'entreprises publiques telles que Becamex. Ce cas d'étude a également permis de mettre en évidence le lien existant entre les éléments locaux de la métropolisation (infrastructures, villes nouvelles, zones industrielles) et ses effets régionaux (formation de corridors 
intrarégionaux). Ce changement d'échelle du développement de cette région urbaine contribue par ailleurs à aggraver le risque de fragmentation territoriale. En l'absence de mécanismes de gouvernance efficaces, les projets menés par les alliances transprovinciales, entrant de plus en plus en compétition avec HCMV, risquent de morceler en profondeur la construction régionale. Les échelles spatiales de ce morcellement sont multiples. Si la métropolisation a déjà aggravé les inégalités entre les districts urbains et les districts ruraux en marge de l'industrialisation, la concurrence interprovinciale empêche les provinces de participer à un projet métropolitain commun. Enfin, le renforcement du pouvoir des provinces économiquement avancées appartenant à la dorsale centrale contribue à marginaliser les provinces périphériques à prédominance rurale de cette région urbaine.

\section{BIBLIOGRAPHIE}

Becamex, 2019. Khu đô thị Tokyu Bình Dương [La zone urbaine nouvelle Tokyu Binh Duong]. [en ligne]. URL: http://becamex.com.vn/vn/linh-vuc-hoat-dong/khu-do-thi/khu-do-thi-tokyu-binhduong (consulté en juillet 2019).

Beresford M., 2008. Doi Moi in Review: the Challenges of Building Market Socialism in Vietnam. Journal of Contemporary Asia, n² 2, p. 221-243.

Binh Duong Statistical Office, 2016. Statistical Yearbook 2016 of Binh Duong province. Thu Dâu Môt, Statistical publishing house, $390 \mathrm{p}$.

Binh Duong Statistical Office, 2017. Statistical Yearbook 2017 of Binh Duong province. Thu Dâu Môt, Statistical publishing house, $391 \mathrm{p}$.

Binh Duong Statistical Office, 2018. Statistical Yearbook 2018 of Binh Duong province. Thu Dâu Môt, Statistical Publishing House, $377 \mathrm{p}$.

Binh Phuoc Statistical Office, 2018. Statistical Yearbook 2018 of Binh Phuoc province. Đông Xoai, Statistical Publishing House, $416 \mathrm{p}$.

Bunnell T., Morshidi S., Barter P.A., 2002. Kuala Lumpur Metropolitan Area. A Globalizing CityRegion. Cities, $\mathrm{n}^{\circ}$ 5, p. 357-370.

Bunnell T., 2004. Malaysia, Modernity and the Multimedia Super Corridor: a Critical Geography of Intelligent Landscapes. Londres/New York, Routledge, $224 \mathrm{p}$.

Deler J-P., Kermel-Torrès D., 2010. Industrialisation et expansion mégapolitaine : le corridor du littoral sud-est de Bangkok. Annales de Géographie, nº 671-672, p. 7-27.

Franck M., 2010. Diffusion spatiale de l'urbanisation et de l'industrialisation et formation d'une région urbaine : le cas de Surabaya, en Indonésie. Annales de Géographie, n 671-672, p. 69-92.

Goldblum C., Franck M., Taillard C., 2012. Villes secondaires et métropolisation en Asie du SudEst. In Goldblum C., Franck M., Taillard C. (dir.). Territoires de l'urbain en Asie du Sud-Est : métropolisations en mode mineur. Paris, CNRS Éditions, p. 267-280. 
Goldblum C., 2015. Territoires de projets : l'Asie orientale à l'épreuve d'un nouveau « régime de production urbaine ». In Franck M., Sanjuan T. (dir.). Territoires de l'urbain en Asie : une nouvelle modernité ? Paris, CNRS Éditions, p. 373-396.

Henriot C., 2013. Villes nouvelles et redéploiement métropolitain à Shanghai. Les nouvelles périphéries urbaines chinoises. Thèse de doctorat en géographie, Université Paris 1 Panthéon-Sorbonne, $441 \mathrm{p}$.

Ho Chi Minh City Statistical Office, 2011. Statistical Yearbook 2011 of Ho Chi Minh City.

Ho Chi Minh City, Statistical publishing house, 345 p.

Ho Chi Minh City Statistical Office, 2015. Statistical Yearbook 2015 of Ho Chi Minh City.

Ho Chi Minh City, Statistical publishing house, 352 p.

Ho Chi Minh City Statistical Office, 2016. Statistical Yearbook 2016 of Ho Chi Minh City.

Ho Chi Minh City, Statistical publishing house, $357 \mathrm{p}$.

Ho Chi Minh City Statistical Office, 2017. Statistical Yearbook 2017 of Ho Chi Minh City.

Ho Chi Minh City, Statistical publishing house, 354 p.

Institut de planification du Sud, 2017. Quy hoạch xây dựng vùng Thành phô Hồ Chí Minh [Schéma directeur de la région métropolitaine de Hô Chi Minh Ville]. Hô Chi Minh Ville, Publications du ministère de la Construction, 505 p.

Centre de recherche sur l'habitat et sur l'immobilier de Binh Duong, 2014. Chương trình phát triễn nhà ở tỉnh Bình Dương đến năm 2020 và định hướng đến năm 2030 [Programme du développement du secteur du logement de la province de Binh Duong jusqu'en 2020 avec des orientations pour 2030]. Thu Dâu Môt, Publications du Comité populaire de Binh Duong, 134 p.

Département des zones économiques, 2017. Báo cáo tổng kêt hoạt động các mô hình khu công nghiệp, khu kinh tê [Rapport sur l'exploitation des modèles de zones industrielles et de zones économiques].

Publications du ministère de la Planification et de l'investissement, 147 p. [en ligne]. URL: http:// www.hapi.gov.vn/News/Download?

url=\%2FUploads\%2FNewsAttachments\%2F01122017101949_4__Bao_cao_tong_ket_mo_hinh_KCN_KKT_30-8-2017_(khong_doi).pdf\&nan (consulté en juillet 2019).

Ville nouvelle de Binh Duong, 2019. Khu công nghệ cao: MapleTree Business Park [La zone de nouvelles technologies : MapleTree Business Park]. [en ligne]. URL: http://

binhduongnewcity.com.vn/vn/chi-tiet-du-an/co-so-kinh-\%20te/overview/khu-cong-nghe-caomaple-tree-business-park (consulté en juillet 2019)

Labbé D., Musil C., 2017. Les « nouvelles zones urbaines » de Hanoi (Vietnam) : dynamiques spatiales et enjeux territoriaux. Mappemonde [En ligne], $\mathrm{n}^{\circ}$ 122. URL: http://

mappemonde.mgm.fr/122as1/

Musil C., Labbé D., Jacques O., 2019. Les zones urbaines « fantômes » de Hanoi (Vietnam). Éclairage sur le (dys)fonctionnement d'un marché immobilier émergent. Cybergeo: European Journal of Geography [En ligne], n 882. https://journals.openedition.org/cybergeo/31466

Nguyen B.T., van der Krabben E., Samura A., 2014. Commercial Real Estate Investment in Ho Chi Minh City: a Level Playing Field for Foreign and Domestic Investors? Habitat International, $\mathrm{n}^{\circ} 44$, p. 412-421.

Pham T.S. et al., 2017. Đề án phát triễn thị trường bất động sản thành phố Hồ Chí Minh giai đoạn 2016-2020, định hướng đên năm 2025 và tầm nhìn đên năm 2030 [Programme de développement du marché immobilier de Hô Chi Minh Ville pour la période 2016-2020, avec les orientations pour 2025 et les perspectives pour 2030]. Hô Chi Minh Ville, Publications du département de la Construction de Hô Chi Minh Ville, $459 \mathrm{p}$. 
Résolution 10/NQ-HĐND/2018, 2018. nghị quyết 10/NQ-HĐND/2018 của Hội đồng nhân dân Thành phố Hồ Chí Minh về chương trình phát triễn nhà ở Thành phố Hồ Chí Minh giai đoạn 2016-2025 [Résolution du Conseil populaire de Hô Chi Minh Ville sur le programme de développement du secteur du logement pour la période 2016-2025].

Somogroup, 2018. Đầu tư dư án bất động sản. Dự án đất nền khu đô thị Cát Lái, quận 2 [Investir dans les projets immobiliers. Projet de zone urbaine nouvelle de Cat Lai (district 2)] [En ligne]. URL: http:// somogroup.vn/bai-viet/3152/dau-tu-du-an-bat-dong-san (consulté en juillet 2019)

Tran K. M., 2019. Des métropolisations en concurrrence : le développement des périphéries urbaines de la région de Hô Chi Minh Ville (Vietnam) sous l'effet de la création de zones industrielles. Thèse de doctorat en géographie, Université Paris 1 Panthéon-Sorbonne, 608 p.

Vietnam Statistical Office, 2016. Statistical Yearbook 2016 of Ho Chi Minh City. Ho Chi Minh City, Statistical publishing house, $946 \mathrm{p}$.

VOV5, 2014. Lể kỷ niệm 25 năm ngày thành lập Tỗng công ty Tân Cảng Sài Gòn [25 ans depuis la création de la société Tân Cang Saigon] [En ligne]. URL: http://vovworld.vn/vi-VN/tin-tuc/le-ky-niem-25nam-ngay-thanh-lap-tong-cong-ty-tan-cang-sai-gon-223168.vov (consulté en juillet 2019).

\section{NOTES}

1. La région du Sud-Est regroupe six provinces situées entre la Côte centrale du Sud et le Delta du Mékong. La région de HCMV est une entité administrative créée par le ministère de la Construction à la fin des années 2000. Au total, la région de HCMV regroupe les six provinces de la région de Sud-Est (Binh Duong, Đông Nai, Ba Ria Vung Tau, Tây Ninh, Binh Phuoc et la municipalité spéciale de HCMV) et deux provinces du Delta du Mékong (Long An et Tiên Giang).

2. Bên Cat est un district situé au centre de Binh Duong. Ce territoire est connu notamment pour son industrialisation rapide et pour la création de la ville nouvelle de My Phuoc.

3. Par rapport aux zones industrielles, les sites industriels s'étendent sur des superficies considérablement moins importantes et sont majoritairement destinés aux entreprises vietnamiennes de taille modeste. La plupart des sites industriels ont été créés à la suite de la réhabilitation et $\mathrm{du}$ regroupement d'usines préexistantes. Le niveau d'organisation et d'équipement des sites industriels est inférieur à celui des zones industrielles.

\section{RÉSUMÉS}

Depuis les réformes du Đổi Mới (Renouveau) de 1986, Hô Chi Minh Ville (HCMV) et les provinces voisines sont devenues le fer de lance de l'économie vietnamienne. Parmi les territoires historiquement dépendants de HCMV, la province de Binh Duong émerge aujourd'hui comme un nouveau pôle métropolitain. Le développement rapide de HCMV et de Binh Duong s'appuie communément sur une industrialisation globalisée, se traduisant par la création de multiples zones industrielles. À partir des années 2000, un secteur immobilier dynamique émerge et transforme en profondeur le paysage et la structure économique locaux. Dans la course à la métropolisation, ces deux provinces ont adopté des stratégies industrielles et urbaines spécifiques, dans l'objectif de renforcer leur compétitivité. En parallèle de la diversification de 
trajectoires urbaines et économiques, de nouvelles dynamiques transprovinciales de complémentarité, de coopération et de concurrence émergent, redéfinissant en profondeur la construction régionale du sud du Vietnam.

Since the Doi Moi (Renovation) reforms initiated in 1986, Ho Chi Minh City (HCMC) and the neighbouring provinces have played a fundamental role in the economic development of Vietnam. Among the neighbouring provinces, that are historically considered as the hinterland of HCMC, Binh Duong is nowadays emerging as a new metropolitan pole. The rapid development of HCMC and Binh Duong is commonly based on a globalized industrialization, which leads to the creation of multiple industrial parks, and an emerging real estate sector, profoundly transforming the local landscape and economic structure. These two provinces have adopted specific industrial and urban strategies in order to strengthen their competitiveness. In parallel with the diversification of urban and economic trajectories, new transprovincial dynamics of complementarity, cooperation and competition appear and redefine in depth the South Vietnam's regional development

\section{INDEX}

Mots-clés : Vietnam, Hô Chi Minh Ville, Binh Duong, industrialisation, urbanisation, mégaprojet urbain, construction régionale

Keywords : Vietnam, Ho Chi Minh City, Binh Duong, industrialization, urbanization, mega urban project, regional development

Thèmes : Sur le Champ - Sur le Terrain

\section{AUTEUR}

\section{KHAC MINH TRAN}

Khac Minh Tran, khacminhtran@gmail.com, est docteur en géographie et attaché temporaire d'enseignement et de recherche à l'Université Paris-Est Marne-la-Vallée. 